Please do not remove this page

RMIT

UNIVERSITY

\title{
Streak generation in wind tunnels
}

Pook, David; Watmuff, Jonathan

https://researchrepository.rmit.edu.au/esploro/outputs/9921859470801341/filesAndLinks?institution=61RMIT_INST\&index=null

Pook, D., \& Watmuff, J. (2014). Streak generation in wind tunnels. Physics of Fluids, 26(7), 1-19.

https://doi.org/10.1063/1.4891180

Document Version: Published Version

Published Version: https://doi.org/10.1063/1.4891180

Repository homepage: https://researchrepository.rmit.edu.au

(c) 2014 AIP Publishing LLC.

Downloaded On 2023/04/26 20:47:26 +1000

Please do not remove this page 
Thank you for downloading this document from the RMIT Research Repository.

The RMIT Research Repository is an open access database showcasing the research outputs of RMIT University researchers.

RMIT Research Repository: http://researchbank.rmit.edu.au/

\section{Citation:}

Pook, D and Watmuff, J 2014, 'Streak generation in wind tunnels', Physics of Fluids, vol. 26, no. 7, pp. 1-19.

See this record in the RMIT Research Repository at:

https://researchbank.rmit.edu.au/view/rmit:24755

Version: Published Version

Copyright Statement: (c) 2014 AIP Publishing LLC

Link to Published Version:

http://dx.doi.org/10.1063/1.4891180 


\section{AIP $\mid$ Physics of Fluids}

\section{Streak generation in wind tunnels}

David A. Pook and Jonathan H. Watmuff

Citation: Physics of Fluids (1994-present) 26, 073605 (2014); doi: 10.1063/1.4891180

View online: http://dx.doi.org/10.1063/1.4891180

View Table of Contents: http://scitation.aip.org/content/aip/journal/pof2/26/7?ver=pdfcov

Published by the AIP Publishing

\section{Articles you may be interested in}

Flows around a cascade of flat plates with acoustic resonance

Phys. Fluids 25, 106104 (2013); 10.1063/1.4825376

A numerical study of the laminar necklace vortex system and its effect on the wake for a circular cylinder Phys. Fluids 24, 073602 (2012); 10.1063/1.4731291

On the spatial linear growth of gravity-capillary water waves sheared by a laminar air flow Phys. Fluids 17, 095101 (2005); 10.1063/1.2033910

Flows past a tiny circular cylinder at high temperature ratios and slight compressible effects on the vortex shedding

Phys. Fluids 15, 1821 (2003); 10.1063/1.1575753

Numerical studies of flow over a circular cylinder at Re $D=3900$

Phys. Fluids 12, 403 (2000); 10.1063/1.870318

\section{A|P| $\left.\right|_{\text {Applied Physics }} ^{\text {Journal of }}$}

Journal of Applied Physics is pleased to announce André Anders as its new Editor-in-Chief 


\title{
Streak generation in wind tunnels
}

\author{
David A. Pook ${ }^{\text {a) }}$ and Jonathan H. Watmuff ${ }^{\text {b) }}$ \\ School of Aerospace, Mechanical and Manufacturing Engineering, RMIT University, \\ Bundoora, Victoria 3083, Melbourne, Australia
}

(Received 28 April 2014; accepted 13 July 2014; published online 31 July 2014)

\begin{abstract}
Computational results are presented which co-validate published experimental observations of steady streaks, deliberately generated by the steady wake of a wire placed upstream of a flat plate with a prescribed leading edge. The largest streak occurs when the wake is generated from a wire placed upstream of the wind tunnel contraction. Normal vorticity passing through the contraction leads to the creation of streamwise vorticity in the test-section via tilting and stretching. The computational results allow the original experiment to be reinterpreted as a receptivity experiment that demonstrates the boundary layer is more receptive to steady streamwise vorticity than normal vorticity. It also suggests an interesting mechanism for the generation of Klebanoff streaks in wind tunnels. The effect of shifting the attachment point at the leading edge on receptivity is also demonstrated. The streak growth is compared to the Optimal streak often used in computational studies. The modal growth of the streak generated by free-stream normal vorticity is found to have a streamwise location of peak energy close to the Optimal streak for wavelengths larger than the leading edge thickness. However, the location of the peak energy for the streamwise vorticity streak varies substantially with wavelength. Differences in wall-normal profiles are also noted. @ 2014 AIP Publishing LLC. [http://dx.doi.org/10.1063/1.4891180]
\end{abstract}

\section{INTRODUCTION}

Steady, spanwise periodic streaks have been used as a model to study bypass transition, the effect of Klebanoff streaks, and the control of transition via Tollmien-Schlichting wave (TS) suppression. Most recent computational studies use the Optimal streak ${ }^{1,2}$ at nonlinear amplitudes ${ }^{3-8}$ Experimental studies have used roughness elements, ${ }^{9-11}$ micro-wings, ${ }^{12-14}$ or more recently vortex generators ${ }^{15,16}$ to create streaks. While these studies have produced streaks with some comparable characteristics to Klebanoff streaks, the disturbance mechanism does not model wind tunnel settling chamber screens which have been empirically linked to Klebanoff streaks. The mechanisms of Goldstein and co-workers ${ }^{17,18}$ describe how free-stream vorticity may create streaks. Further, computational results have shown that a steady streak generated from a Squire mode through free-stream forcing can amplify TS growth at low to moderate amplitudes and exhibit a secondary instability threshold significantly lower than the Optimal streak. ${ }^{19}$ This result indicates the importance of accurately modeling a streak for transition prediction. Transient/algebraic/non-modal growth theories explain how large streaks can grow in a boundary layer but accurate prediction requires a link with free-stream disturbances.

The studies of Kogan et al. ${ }^{20}$ and Watmuff ${ }^{21}$ used a thin wire upstream of the leading edge to create a streak in the downstream layer. In one particular case, Watmuff ${ }^{21}$ located the wire upstream of the wind tunnel contraction. This created a very strong streak yet the wire wake magnitude was nearly immeasurable. This case provides a starting point to study how Klebanoff streaks are related to wind tunnel screens.

\footnotetext{
a) Electronic mail: d.pook.engineer@gmail.com

b) Electronic mail: jon.watmuff@ rmit.edu.au
} 


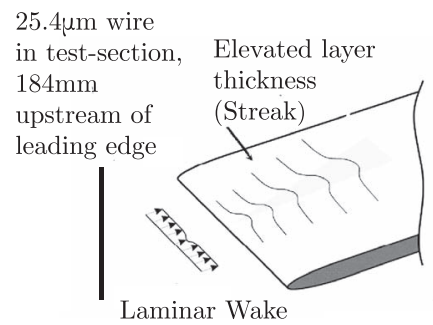

(a)

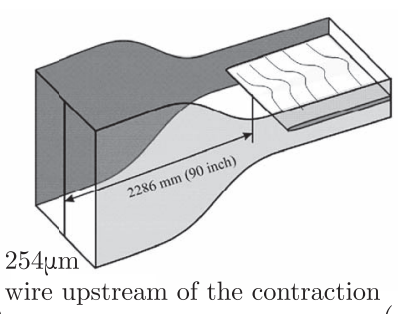

(b)

wire upstream of the contraction

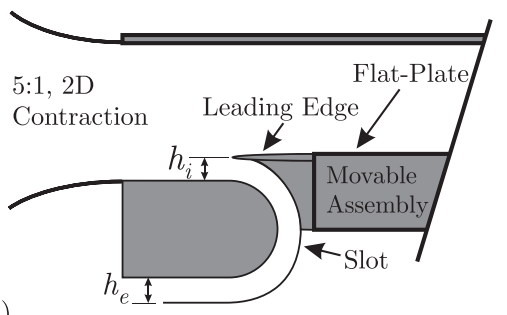

FIG. 1. (a) Wire in the test-section, Watmuff ${ }^{21}$ case 2 T. (b) Wire upstream of the contraction, Watmuff ${ }^{21}$ case $4 U$. (c) Test-section of Watmuff. ${ }^{21}$

The aim of this work is to computationally reproduce the experiment of Watmuff. ${ }^{21}$ Two wire configurations are considered, as depicted in Fig. 1. In the first configuration, the wire was placed in the test-section upstream of the leading edge. This configuration is labeled case $2 \mathrm{~T}$ in accordance with the original paper. The original experiment tested wires with differing diameters, hence the numerical designation. In the second configuration, the wire was located upstream of the wind tunnel contraction. The configuration is labeled case $4 \mathrm{U}$. In both configurations, the wire was normal to the free-stream and the leading edge. The case $2 \mathrm{~T}$ wire produced an order of magnitude stronger wake but an order of magnitude weaker streak. The reason was unexplained. To reproduce the experimental results with Computational Fluid Dynamics (CFD), it will be shown that factors originally not identified are crucial. The resultant streaks will be documented and a comparison made to the Optimal streak. The CFD results will show the Watmuff experiment provides experimental evidence that the boundary layer is more receptive to steady streamwise vorticity than normal vorticity, as found in the recent computations of Schrader et al. ${ }^{22}$ The results will indicate how settling chamber screens may create Klebanoff streaks by the tilting and stretching of vorticity in a wind tunnel contraction.

\section{CFD MODELING OF THE TEST-SECTION}

\section{A. Watmuff test-section experimental configuration}

A sketch of the Watmuff ${ }^{21}$ wind tunnel test-section is shown in Fig. 1(c). The height of the leading edge $\left(h_{i}\right)$ could be adjusted and the flow attachment on the leading edge altered by adjusting the exit height $\left(h_{e}\right)$ of the slot. Increasing $h_{e}$ would move the attachment point to the topside of the leading edge. Wool tuft visualization showed the flow in the exit slot was steady and attached. The leading edge attachment point was unknown. It will be demonstrated that to reproduce the experimental results requires modeling of the test-section and contraction. The CFD model uses the same leading edge geometry described by

$$
\left(\frac{3 x}{160 h}\right)^{4}+\left(\frac{y}{h}\right)^{2}=1
$$

on the topside and

$$
\left(\frac{3|x|}{160 h}\right)^{0.205}+\left(\frac{-3 y}{16 h}\right)^{2}=1
$$

on the lowerside for $x=[-203.2,0] \mathrm{mm}$. The topside is a super-ellipse with an aspect ratio of 53.3 and a thickness $(h)$ of $3.81 \mathrm{~mm}$. The leading edge spanned the $1 \mathrm{~m}$ wide test-section and was positioned at $h_{i}=43.5 \mathrm{~mm}(11.5 h), 470 \mathrm{~mm}$ downstream from the exit of the 5:1, two-dimensional contraction. The Reynolds number based on the leading edge thickness is 2500 .

\section{B. CFD two-dimensional base-flow}

The spanwise uniform layer, unaffected by a streak, is modified by the leading edge attachment position. The effect of altering the slot exit height, $h_{e}$, is modeled in the CFD simulations by altering 


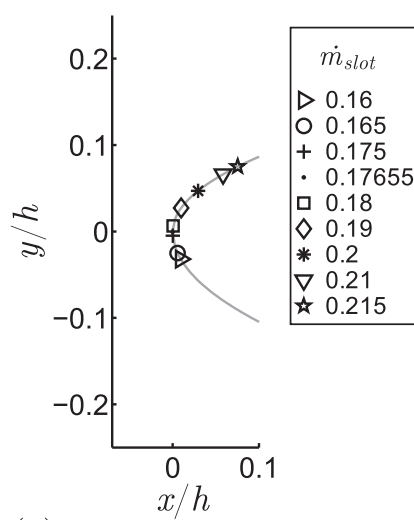

(a)

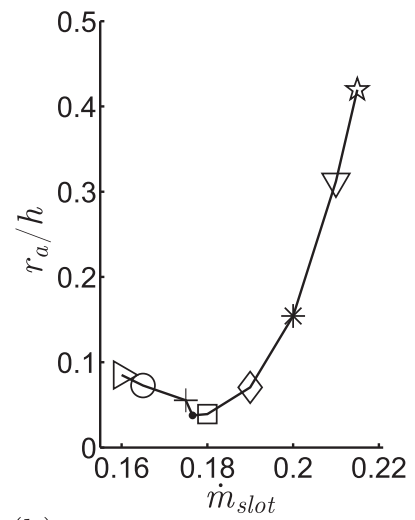

(b)

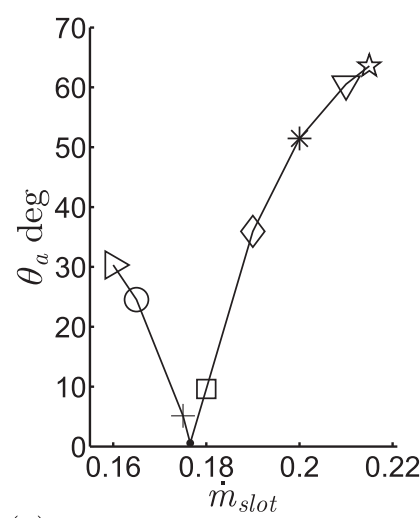

(c)

FIG. 2. (a) Leading edge attachment position with varying slot massflow, $\dot{m}_{\text {slot }}$, calculated with CFD. Markers indicate $\dot{m}_{\text {slot }}$. (b) Radius of curvature $\left(r_{a}\right)$ at attachment. (c) Tangent angle of the leading edge surface $\left(\theta_{a}\right)$ at attachment.

the ratio of the massflow exiting through the slot outflow boundary condition relative to that over the plate. Attachment on the leading edge centerline is achieved with a slot massflow ratio $\left(\dot{m}_{\text {slot }}\right)$ of 0.17655 . The slot massflow remained constant during the Watmuff experiment but its value was unknown.

Figure 2(a) shows that increasing the slot massflow shifts the attachment point to the topside of the leading edge. Figure 2(b) shows the body radius-of-curvature at attachment $\left(r_{a}\right)$ is a minimum when the flow attaches on the leading edge centerline. The angle between the normal line at attachment and the undisturbed free-stream $\left(\theta_{a}\right)$ is also shown in Fig. 2(c).

For a constant, far-upstream inflow condition $\left(U_{\infty}\right)$, the boundary-layer edge velocity will be modified by the changing slot massflow. Boundary layer parameters are calculated with local edge velocities, defined as the $99 \%$ of the maximum wall-tangent velocity on a profile in the wall-normal direction $\left(U_{\text {edge }}\right)$. Figure 3 shows that the streamwise development of displacement thickness $\left(\delta^{*}\right)$ is relatively unaffected by slot massflow, maintaining the Blasius growth rate (1.72) downstream. The virtual origin for all slot massflows is small and neglected. The end of leading edge curvature (start of the flat-plate) occurs at $R=370$ for the $\dot{m}_{0.17655}$ slot massflow, where $R$ is the square root of the Reynolds number based on streamwise distance. The pressure coefficient $\left(C_{p}\right)$ defined as

$$
C_{p}=\frac{2\left(p-p_{r e f}\right)}{\rho U_{\infty}^{2}},
$$

is also shown in Fig. 3. From $R=600$ to the end of the plate near $R \approx 1500$, the pressure coefficient decreases $0.4 \%$. Attachment on the topside of the leading edge reduces, and can eliminate, the adverse pressure gradient after attachment. Unpublished pressure measurements in the leading edge

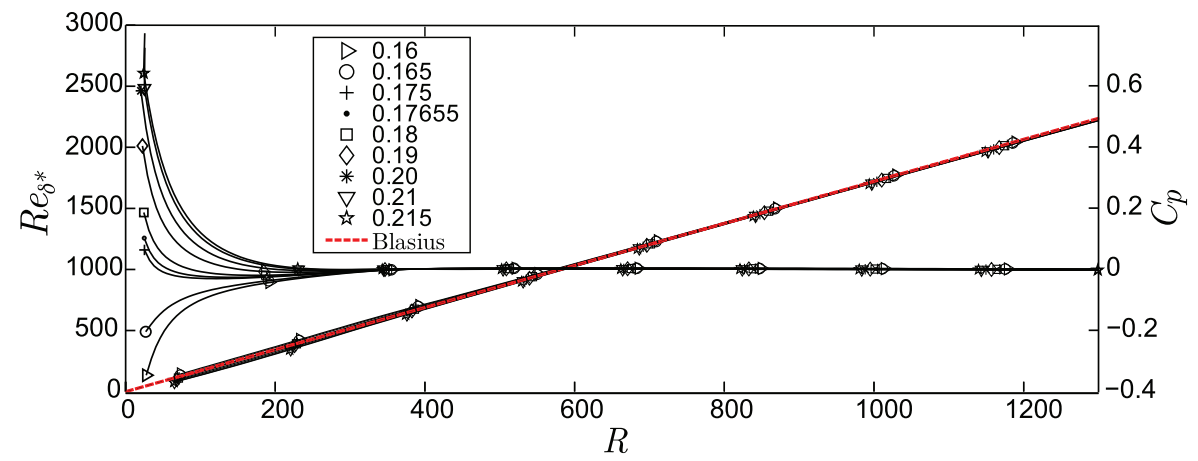

FIG. 3. $R e_{\delta^{*}}$ versus $R$ and $C_{p}$ versus $R$ for varying slot massflow. 

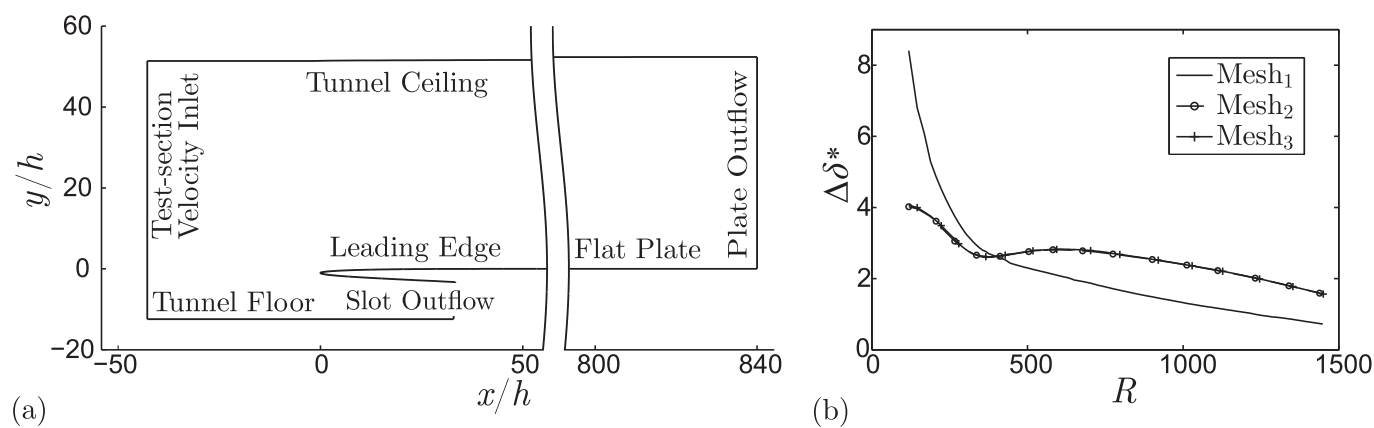

FIG. 4. (a) Test-section boundary conditions. (b) Effect of mesh refinement on $\Delta \delta *$ for the case $2 \mathrm{~T}, \dot{m}_{0.17655}$ streak.

region by Watmuff revealed no adverse pressure gradient, indicating topside attachment on the leading edge.

\section{MESH AND CFD METHODS}

The Ansys Fluent CFD package is used for all calculations. The pressure-based, coupled solver is used with second-order upwind differencing for pressure and third-order Monotonic UpstreamCentered Scheme for Conservation Laws (MUSCL) momentum differencing. All simulations are steady. The CFD simulations are divided into two separate mesh regions: the test-section region (contains leading edge, slot, and plate); and contraction region.

\section{A. Test-section mesh}

Data in the test-section boundary layer are reported in terms of a body-fitted coordinate system, i.e., streamwise ( $x$ direction, $U$ velocity) implies tangent to the leading edge/flat-plate with the origin at the tip of the leading edge, wall-normal ( $y$ direction, $V$ velocity), and spanwise ( $z$ direction, $W$ velocity).

Figure 4(a) shows an overview of the test-section mesh boundary conditions. The mesh in the boundary layer is composed of structured, highly orthogonal, quadrilateral control volumes (CV). The streamwise and wall-normal growth of $\mathrm{CV}$ spacing is $\sim 2 \%$. The $\mathrm{CV}$ in the spanwise direction are clustered towards the streak centerline. The mesh resolution is halved outside the boundary layer with the use of hanging nodes. No-slip walls are employed on the test-section ceiling and floor with a coarse mesh $(\sim 10 \mathrm{CV}$ in the layer) as they are not expected to influence results. The tunnel ceiling is contoured to provide a minimal pressure gradient by (approximately) growing with the Blasius boundary layer. At the start of the leading edge, the ceiling is located $241.3 \mathrm{~mm}$ above the test-section floor. The slot and plate outflows are specified as outflow boundary conditions with prescribed massflows.

A symmetry condition is utilized in the spanwise ( $z$ ) direction on the wake/streak centerline to reduce the mesh size. The span of the domain, including symmetry, is $80 \mathrm{~mm}$ for case $2 \mathrm{~T}$ and 200 $\mathrm{mm}$ for case $4 \mathrm{U}$. The case $2 \mathrm{~T}$ test-section mesh has $160 \mathrm{CV}$ in the $z$-direction. The case $4 \mathrm{U}$ mesh has $200 \mathrm{CV}$ uniformly spaced.

The wake simulating the case $2 \mathrm{~T}$ wire is imposed on the spanwise $(z)$ invariant base-flow at the inlet of the test-section mesh, $163 \mathrm{~mm}(42.8 h)$ upstream of the leading edge. The base-flow velocity distribution across the test-section inlet (tunnel floor to ceiling) is obtained from a two-dimensional simulation with both the contraction and test-section meshes joined. This allows for any upstream influence of slot massflow variation. A wake simulating the case $4 \mathrm{U}$ streak is imposed on the testsection mesh $400 \mathrm{~mm}$ upstream of the leading edge (105h). This extra inlet distance is created by extending the test-section mesh upstream. 
TABLE I. Mesh resolution for a grid refinement study using the case2T streak.

\begin{tabular}{lccc}
\hline \hline & $\begin{array}{c}\text { Streamwise CV } \\
\text { leading edge, flat-plate }\end{array}$ & $\begin{array}{c}\text { Wall-normal CV in } \\
\text { boundary layer }\end{array}$ & Spanwise CV \\
\hline Mesh $_{1}$ & 125,175 & $\sim 15$ & 40 \\
Mesh $_{2}$ & 250,350 & $\sim 30$ & 80 \\
Mesh $_{3}$ & 500,700 & $\sim 60$ & 160 \\
\hline \hline
\end{tabular}

\section{B. Test-section mesh refinement}

A mesh refinement study is performed for the case2T streak with a slot massflow $\dot{m}_{0.17655}$. Mesh resolutions are listed in Table I. Each mesh is refined in the leading-edge/plate boundary layer region by doubling the number of $\mathrm{CV}$ in all directions. The primary variable of interest to compare with experiment is the spanwise variation of displacement thickness defined by

$$
\Delta \delta^{*}=\frac{\delta_{\text {max }}^{*}-\delta_{\min }^{*}}{\delta_{\text {base }}^{*}},
$$

at a given streamwise position. Figure 4(b) shows $\Delta \delta^{*}$ has minimal variation between $\mathrm{Mesh}_{2}$ and Mesh $_{3}$. Mesh 3 will be used for the remainder of this work. A grid convergence study is not performed for case $4 \mathrm{U}$. Comparison to experimental data will be used for validation.

\section{Contraction mesh}

Figure 5(a) shows the contraction mesh boundary conditions. The mesh is composed of structured, near-orthogonal, quadrilateral CV concentrated near the floor of the contraction. A symmetry plane is used in the spanwise $(z)$ direction and on the contraction centerline ( $y$ direction). Two hundred $\mathrm{CV}$ are used in the $y$-direction, $440 \mathrm{CV}$ in the streamwise direction with uniform spacing in the contraction, and $160 \mathrm{CV}$ in the $z$ direction, for a total mesh size of $14.5 \times 10^{6} \mathrm{CV}$. The inlet is $150 \mathrm{~mm}$ upstream of the contraction floor curvature that begins at $x=0 \mathrm{~mm}$ and ends at $x=1524 \mathrm{~mm}$. This defines the length of the contraction, $L_{c}$. The contraction floor between these limits is described by the polynomial $(x$ in $\mathrm{m})$,

$$
y=0.35222 x^{5}-1.34196 x^{4}+1.36343 x^{3}-0.5299 .
$$

The outlet of the mesh would extend past the start of the leading-edge $\left(x_{l e}=1994 \mathrm{~mm}\right)$ were it included. Wake samples for the test-section inlet boundary condition are taken $70 \mathrm{~mm}$ downstream from the end of the contraction curvature $\left(x_{t s b c}\right)$ where there is a minimal pressure gradient. Both noslip and slip-wall boundary conditions will be used for the contraction floor to explore the influence of the contraction boundary layer. The inlet velocity profile is assumed uniform $\left(1.95 \mathrm{~ms}^{-1}\right)$ in the $y$-direction.
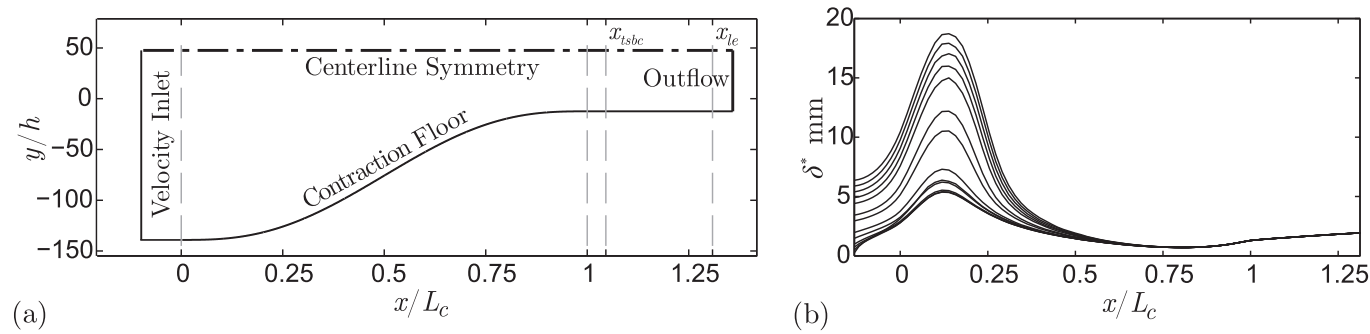

FIG. 5. (a) Contraction mesh boundary conditions. (b) The effect of contraction floor boundary layer thickness ( $\delta *)$ entering contraction on the layer thickness at the exit. 


\section{Two-dimensional flow through the contraction}

The experiment of Watmuff ${ }^{21}$ did not measure the boundary layer exiting the contraction. The proximity of the test-section leading edge to the tunnel floor suggests the layer thickness may be an issue. However, two-dimensional CFD results shown in Fig. 5(b) reveal the displacement thickness of the boundary layer exiting the contraction is independent of the layer thickness entering the contraction, indicating it is not an important parameter. A uniform velocity profile into the contraction is a sufficient boundary condition.

\section{E. Development of the wire wake}

The laminar wake of a wire can be predicted analytically by a linear diffusion equation derived from the Navier-Stokes equations. ${ }^{23}$ The velocity decrement $(\Delta U)$ can be expressed as

$$
\frac{\Delta U}{U_{\infty}}=C_{d} \sqrt{\frac{R e_{d} d}{16 \pi x}} \exp \left(-\frac{U_{\infty} z^{2}}{4 v x}\right),
$$

where $d$ is the wire diameter, $C_{d}$ is the wire drag coefficient, and $R e_{d}$ is the wire Reynolds number based on diameter. The spanwise velocity component $(W)$ can be derived from continuity as

$$
\frac{W}{U_{\infty}}=C_{d} z \sqrt{\frac{R e_{d} d}{64 \pi x^{3}}} \exp \left(-\frac{U_{\infty} z^{2}}{4 v x}\right) .
$$

The relationship between $C_{d}$ and $R e_{d}$ found by Sen $e t a l .{ }^{24}$ is used to model the wire $C_{d}$,

$$
C_{d}=0.26+7.89 R e_{d}^{-0.5} \text { for } 15 \leq R e_{d} \leq 40 .
$$

Equations (6) and (7) are used to create the CFD inlet boundary condition. For case2T, the wire diameter is $25.4 \mu \mathrm{m}$ and $C_{d}=2.25$. The wire is located $7250 d$ upstream of the leading edge. For case $4 \mathrm{U}$, the wire diameter is $254 \mu \mathrm{m}$ and $C_{d}=1.62$. The wire is located $9000 d$ upstream of the leading edge, and upstream of the contraction.

Figure 6(a) compares the streamwise velocity component of the CFD wake with the measurements of Watmuff. ${ }^{21}$ The case $2 \mathrm{~T}$ wake agreement is very good. For case $4 \mathrm{U}$, the agreement in width is good but the CFD predicted magnitude is half that measured experimentally. The strength of the experimental wake is $0.1 \% U_{\infty}$ which was considered difficult to measure by Watmuff as it is only slightly greater than the background variation. It will be shown the case $4 \mathrm{U}$ wake has been predicted with sufficient accuracy.

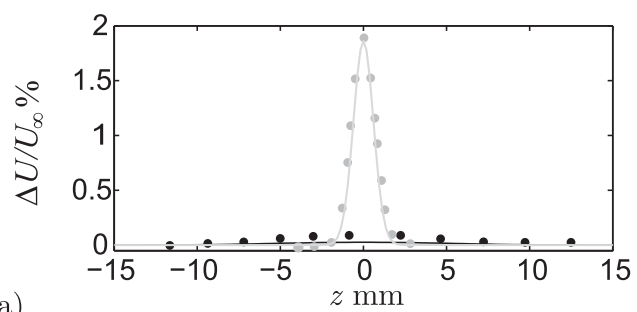

(a)
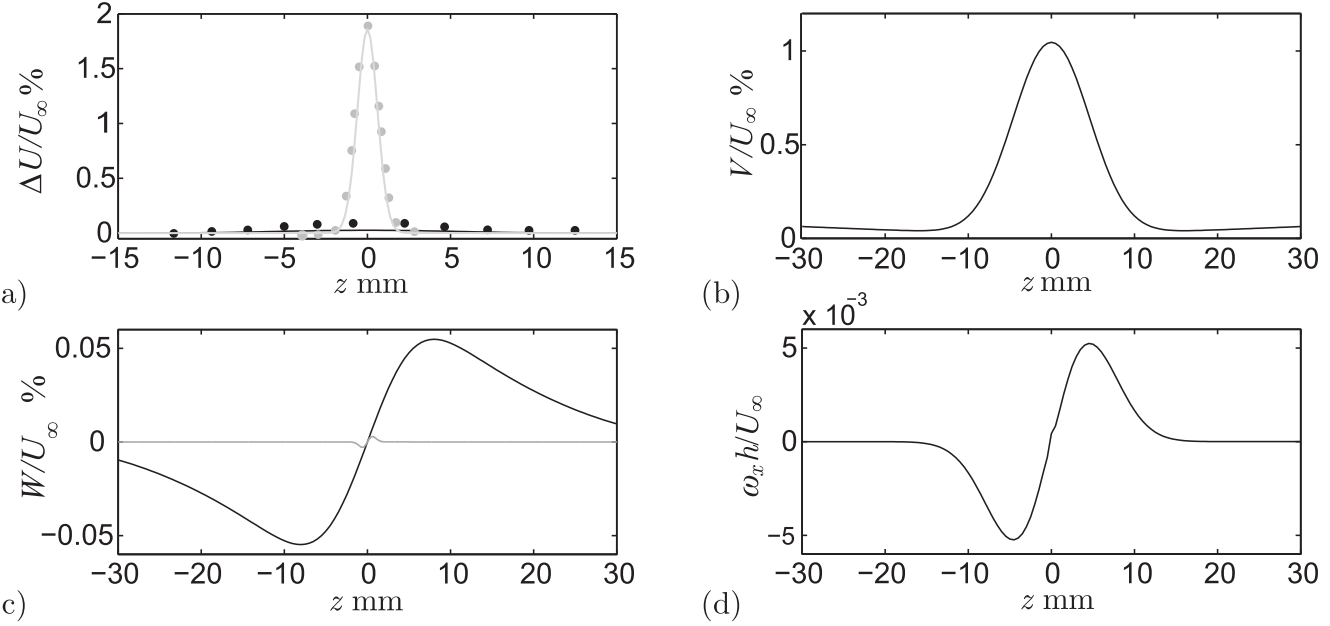

(b)

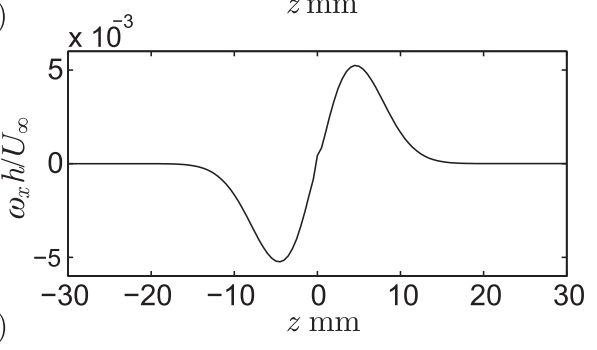

FIG. 6. Spanwise wake profile, $63.5 \mathrm{~mm}(16.7 \mathrm{~h})$ upstream of the leading edge. Grey is case2T. Black is case $4 \mathrm{U}$. Circle markers are experimental measure of Watmuff ${ }^{21}\left(\Delta U / U_{\infty}\right.$ only). $V$ and $\omega_{x}$ shown for case $4 \mathrm{U}$ only. 

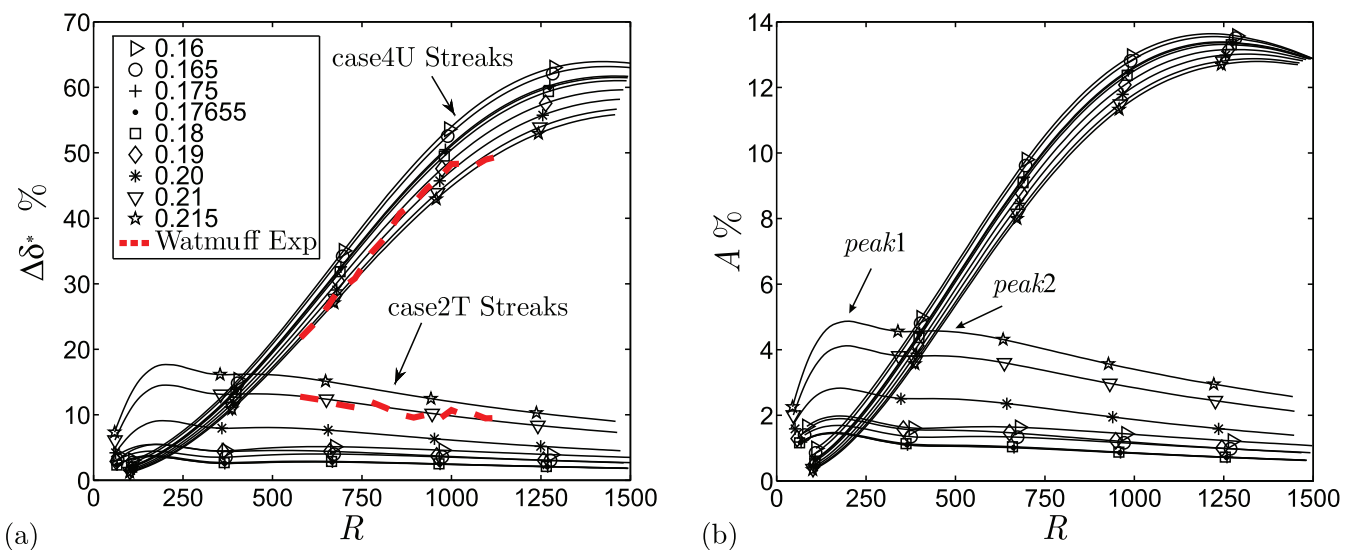

FIG. 7. (a) Streamwise development of the case $2 \mathrm{~T}$ and case $4 \mathrm{U}$ streak strength for varying slot massflow, compared with the experiment of Watmuff. ${ }^{21}$ (b) Streamwise development of the case2T and case $4 \mathrm{U}$ streak strength for varying slot massflow.

\section{STREAK STRENGTH AND AMPLITUDE}

Watmuff $^{21}$ observed the case2T wire would produce a steady laminar wake in the free-stream with $\Delta U / U_{\infty}=1.8 \%$ and a half-width of $2 \mathrm{~mm}$ when measured $63.5 \mathrm{~mm}$ upstream of the leading edge. The wake was composed primarily of vorticity aligned normal to the flat-plate. A streak in the downstream plate boundary layer developed with a peak strength, defined by (4), of $\Delta \delta^{*}=12 \%$ at $R \approx 600$. The case $4 \mathrm{U}$ wire produced a wider but near immeasurable wake upstream of the leading edge. However, the peak streak strength measured was $\Delta \delta^{*}=50 \%$ at $R=1200$.

\section{A. Case2T: Wire in the test-section}

Initial attempts to reproduce the case $2 \mathrm{~T}$ experimental result using CFD grossly underestimated the streak strength, even though the CFD wake closely matched the experimental wake (see Fig. 6(a)). Initial attempts assumed attachment on the leading edge centerline $\left(\dot{m}_{0.17655}\right)$. The streak that developed exhibited a strength of approximately $\Delta \delta^{*}=2.5 \%$, an order of magnitude weaker than observed by Watmuff. ${ }^{21}$

Figure 7(a) shows that adjusting the slot massflow so attachment occurs away from the leading edge centerline significantly increases the streak strength. The agreement with the case $2 \mathrm{~T}$ experimental data is good when the slot massflow is $\dot{m}_{0.21}$. Topside attachment increases the stretching of the normal vorticity that is turned into streamwise vorticity via the mechanism of Goldstein et al. ${ }^{17}$ For each slot massflow, two streamwise maxima of streak strength can be seen. The easily discernible and larger upstream peak is called peak 1 , the more moderate downstream peak in the vicinity of $R$ $\simeq 500$, peak 2 .

Figure 7(b) shows streak growth in the more commonly used measure, streak amplitude $(A)$ defined on a given $y z$ plane as

$$
A=\frac{\max \left[U(y, z)-U_{2 D}(y)\right]-\min \left[U(y, z)-U_{2 D}(y)\right]}{2 U_{\infty}} .
$$

The amplitude measure is related to the difference between the maximum and minimum streamwise velocity deviation on a cross-stream plane. The amplitude measure shows qualitative agreement with $\Delta \delta^{*}$ and two streamwise maxima are seen for the case2T streaks. A maximum streak amplitude of approximately $A=5 \%$ is obtained for the $\dot{m}_{0.215}$ slot massflow. This amplitude is well below published thresholds for streak secondary instability in a zero pressure gradient ${ }^{3,19}$ and is likely to have a minimal affect on TS growth. ${ }^{6,7}$ The strength and amplitude measures are not linearly related for the streaks considered. The ratio $\Delta \delta * / A$ varies with streamwise position and streak strength, 
between 2.3 and 3.8. The ratio increases with increasing streak strength but is nearly constant in the flat-plate region for a given streak.

\section{B. Case4U: Wire upstream of the contraction}

Initial CFD attempts failed to reproduce the case $4 \mathrm{U}$ streak using a free-stream wake composed only of $U$ and $W$ components that matched the experimental data. The resulting streak was near immeasurable, indicating neither the streamwise or crossflow velocity component of the wake is responsible.

To resolve this anomaly, the wake of the wire upstream of the contraction is estimated using (6) and (8). The two-dimensional wake is then imposed at the inlet boundary condition of the contraction mesh. The $\Delta U$ component of the wake exiting the contraction is shown in Fig. 6. It is approximately half the experimentally measured wake strength. Not measured in the experiment were the $V$ and $W$ components, which are also shown in Fig. 6 with the streamwise vorticity $\left(\omega_{x}\right)$. The largest velocity component of the wake is the $V$ component. Its maximum amplitude is approximately $1 \%$ of the free-stream velocity. The wake downstream of the contraction can be considered as primarily streamwise vorticity.

Figure 7(a) shows the strength of the case $4 \mathrm{U}$ streak when including the wake $V$ component. Topside attachment on the leading edge reduces the strength of the case $4 \mathrm{U}$ streak but increases the strength of the case2T streak. The opposite influence of the attachment location allows the

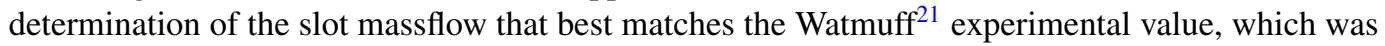
set by the slot exit height (Fig. 1(c)) and was held constant during the experiment. A good match is observed in Fig. 7(a) between experiment and CFD for the slot massflow $\dot{m}_{0.21}$. The closely matching streamwise development of streak strength for both case $2 \mathrm{~T}$ and case $4 \mathrm{U}$ provides convincing evidence that the CFD has closely matched the experiment.

The case 4 U streak amplitude is shown in Fig. 7(b) and is seen to peak at a value below that for a secondary instability of the Optimal streak. ${ }^{3}$ However, it could be strong enough to alter TS growth rates significantly. ${ }^{6,7}$

The case $4 \mathrm{U}$ maximum streak strength occurs downstream of the peak amplitude but the general trends are the same. The streak growth is slow initially, increasing to a near constant linear growth beyond $R \approx 250$, until reaching a maximum at $R \approx 1500$. The reduced growth in the early layer is consistent with observations of Klebanoff streak $u_{r m s}$ growth by Fransson et al. ${ }^{25}$ and the downstream linear growth is consistent with Westin et al. ${ }^{26}$ Unlike the case2T streak, the minimum strength does not occur for attachment on the centerline, $\dot{m}_{0.176555}$. Rather, it occurs for the attachment on the topside, furthest from the leading edge centerline. However, the sensitivity to attachment is significantly reduced relative to case $2 \mathrm{~T}$. This is consistent with the experimental observations that the leading edge does not affect Klebanoff streaks, ${ }^{27,28}$ and the analysis of Goldstein et al. ${ }^{18}$ that considers the leading edge to be infinitely thin when studying the receptivity to free-stream streamwise vorticity.

\section{THE EFFECT OF LEADING EDGE RADIUS AT ATTACHMENT FOR CASE2T}

The large change in case2T streak strength with attachment position can be ascribed to the effective change in leading edge bluntness. Ustinov ${ }^{29}$ predicts the amplitude of a streak generated by normal vorticity should scale almost linearly with leading edge radius and be weakly dependent on spanwise scale. Schrader $e t$ al. $^{22}$ found an approximate factor of three increase in streak growth (linear amplitudes) when changing the leading edge aspect ratio by a factor of 3.3. The radius of curvature at attachment for the $\dot{m}_{0.17655}$ and $\dot{m}_{0.215}$ slot massflows varies by a factor of 8 (see Fig. 2 ), while the change in $\Delta \delta^{*}$ at peak 1 is a factor of 4.9. The change in amplitude $(A)$ is only a factor of 3.4. Figure 8(a) shows the ratio of streak amplitude to leading edge radius at attachment $\left(\frac{A}{r_{a}}\right)$ for the two peak positions. The ratio is nearly constant when the attachment point is on the lowerside of the leading edge. However, on the topside the ratio reduces significantly with attachment position. The scaling with the radius of curvature at attachment is nonlinear and streamwise dependent for the streaks considered. 


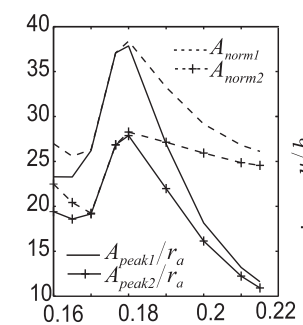

(a)

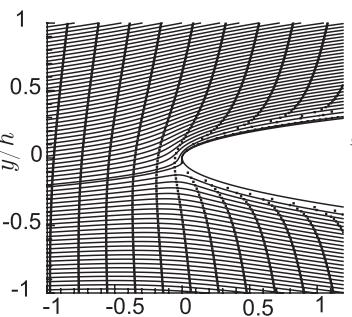

(b)

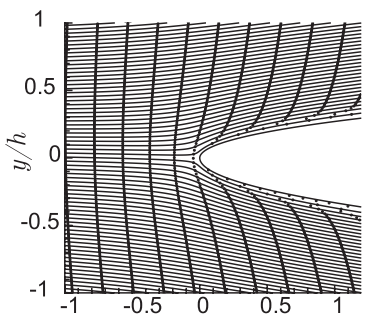

(c)

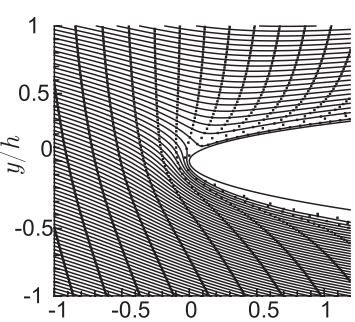

(d)

FIG. 8. (a) case2T scaled streak amplitude at peak1 and peak2. (b) Streamlines with particles approaching the leading edge, $\dot{m}_{0.16}$, (c) $\dot{m}_{0.17655}$, (d) $\dot{m}_{0.215}$.

Figure 8 reveals that the likely cause of the nonlinear scaling of streak amplitude with the radius of curvature at attachment is the tilting of streamlines approaching the leading edge. Markers on the streamlines denote particles released on a vertical line $14 h$ upstream of the leading edge at intervals of $0.025 \mathrm{~s}$. A line joining markers released at the same instant approximates a material line of normal vorticity (wake) convecting in an inviscid flow. The spread of particles in the attachment region corresponds to the vortex stretching mechanism described by Goldstein et al. ${ }^{17}$ Increased stretching is seen when the attachment point shifts away from the centerline, but the material line is also rotated relative to the streamlines. This rotation reduces the component of vorticity parallel to attachment surface that can be stretched. Significantly, greater rotation is achieved with the topside attachment. Assuming the material line maintains its undisturbed free-stream orientation (vertical), then the component of vorticity to be stretched is dependent on the body angle at the attachment position relative to the undisturbed streamline $\left(\theta_{a}\right.$ see Figure 2$)$. A scaled streak amplitude $\left(A_{\text {norm }}\right)$ may then be defined as

$$
A_{\text {norm }}=\frac{A_{\text {peak }}}{r_{a} \cos \left(\theta_{a}\right)}
$$

Figure 8(a) shows $A_{\text {norm }}$ at peak1, and peak 2 provides a more constant case2T streak amplitude, particularly for the topside attachment slot massflows at peak2. However, the simple normalization neglects pressure gradients and the streamwise vorticity component generated by the effective rotation.

\section{WIRE WAKE THROUGH THE CONTRACTION}

The case $4 \mathrm{U}$ streak is generated by receptivity to streamwise vorticity. The initial wake from the wire upstream of the contraction is described by (6) with the $C_{d}$ estimated as 1.62 by (8). This wake can be considered as normal vorticity prior to the contraction, i.e., oriented in the direction of the geometrical contraction. Inside the contraction the normal vorticity is tilted relative to the streamlines. This introduces a streamwise oriented component of vorticity which is then stretched by the accelerating flow.

Figure 9 depicts the tilting and stretching process based on the assumption of inviscid flow carrying an initial normal vorticity component. A material line represents a vortex tube and at the contraction inlet the material line is normal to the flow. As the material line convects downstream, it can be seen to tilt due to the flow accelerating more rapidly on the contraction centerline. The tilting introduces a component of streamwise oriented vorticity. As the material line convects further it is stretched. Conservation of angular momentum requires this stretching to increase the vorticity. Significant stretching does not occur until halfway through the contraction. The maximum tilting and stretching occurs just prior to the contraction exit where the material line is increasingly streamwise orientated away from the contraction centerline. ${ }^{30}$

The leading edge of Watmuff ${ }^{21}$ was located $47.3 \mathrm{~mm}(12.4 h)$ above the contraction floor at $x / L_{c}=1.3$. Figure 9 predicts the wake would be composed primarily of streamwise vorticity at this 


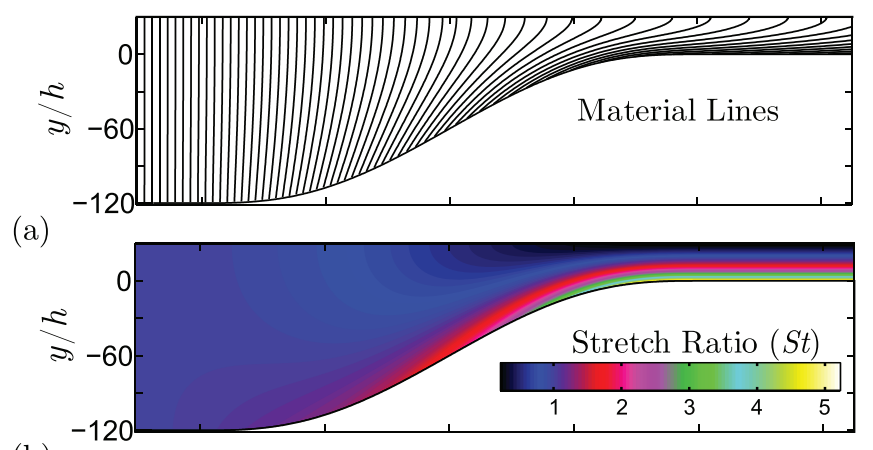

(b)

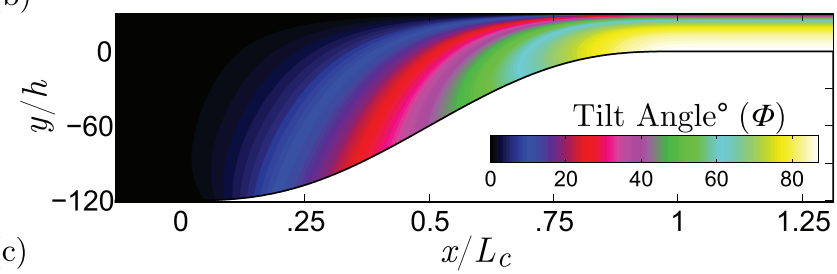

FIG. 9. Stretching and tilting of a material line through the 5:1, two-dimensional contraction, lower half shown. Inviscid flow. $L_{c}$ is the contraction length $(1524 \mathrm{~mm})$. (a) Material lines. (b) Stretching ratio. (c) Tilt angle.

position. An estimate of the streamwise vorticity can be formulated as

$$
\omega_{x}=\omega_{y \text { in }} \times S t \times \sin (\phi),
$$

where $\omega_{\text {yin }}$ is the uniform normal vorticity entering the contraction, $S t$ is the stretching ratio, and $\phi$ is the tilt angle at the position of interest. The estimated maximum streamwise vorticity at the leading edge is $\omega_{y \text { in }} \times 1.68 \times \sin \left(83^{\circ}\right)=1.67 \omega_{y \text { in }}$. The predicted streamwise vorticity exiting the contraction is significantly greater than the normal vorticity that enters. However, the estimate neglects viscous effects.

Figure 10 shows contours of streamwise vorticity on a cross-stream plane $11 \mathrm{~h}$ upstream of the leading edge. Two simulations are shown. The first includes the contraction boundary layer (no-slip wall). The second uses a slip-wall boundary condition for the contraction floor, preventing a contraction boundary layer. Inclusion of the contraction layer produces relatively stronger cores of vorticity about $4.5 \mathrm{~h}$ above the contraction floor. A secondary core of opposite rotation and weaker strength is located in the floor boundary layer. The peak streamwise vorticity strength is $97 \%$ of the peak normal vorticity entering the contraction $\left(\max \left(\omega_{\text {yin }}\right)\right)$. The peak normal vorticity entering the

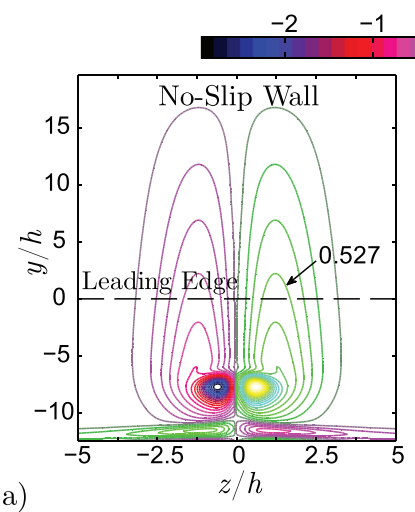

$$
\text { (b) }
$$
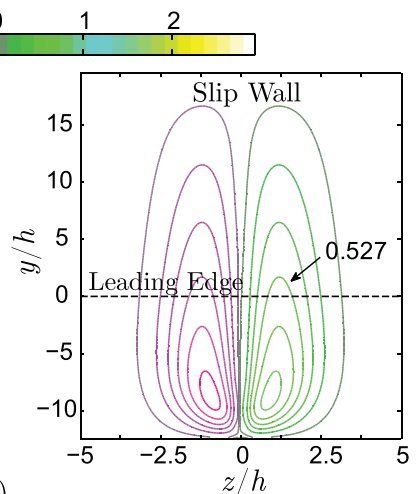

FIG. 10. Comparison of no-slip and slip-wall contractions on a cross-sectional plane $11 \mathrm{~h}$ upstream of leading edge, $x / L_{c}=$ 1.28 in Fig. 9. (a) No-slip wall contraction. Contours of $\omega_{x} / \max \left(\omega_{y \text { in }}\right), 40$ levels from -2.9 to 2.9. Level at 0.527 marked. (b) Slip-wall contraction, same levels. (c) $\omega_{x} / \max \left(\omega_{y \text { in }}\right)$ on spanwise profile at leading edge height, $11 \mathrm{~h}$ upstream. 


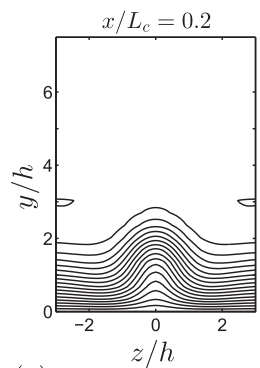

(a)
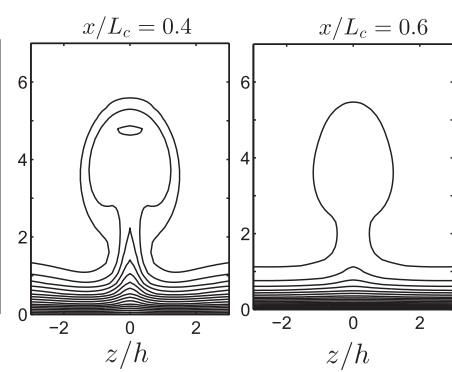

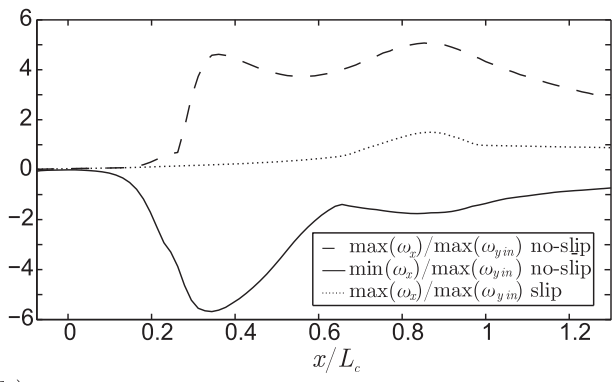

(b)

FIG. 11. (a) Contours of streamwise velocity in the contraction on vertical planes. 20 levels. The contraction wall is at $y / h$ $=0$. (b) Maximum and minimum streamwise vorticity $\left(\omega_{x}\right)$ through the contraction, scaled by the maximum normal vorticity into the contraction $\left(\max \left(\omega_{y i n}\right)\right)$.

contraction for case $4 \mathrm{U}$ is $\max \left(\omega_{\text {yin }}\right)=22.2 \mathrm{~s}^{-1}$. Vorticity cores are not visible when using a slip wall.

Despite the significant difference in the flow pattern for the slip/no-slip wall contractions, Fig. 10(c) shows the streamwise vorticity on a spanwise profile at the leading edge height is almost identical. The maximum streamwise vorticity on the profile is $60 \%$ of the peak normal vorticity entering the contraction, substantially less than the inviscid prediction. However, the strength of the case $4 \mathrm{U}$ streak indicates this is a significant amount of streamwise vorticity. The maximum local flow angle on the spanwise profile is $0.5^{\circ}$. More importantly, the maximum spatial gradient of the flow angle near the leading edge is about $65^{\circ} \mathrm{m}^{-1}$. This is significantly greater than allowed by the National Wind Tunnel Complex (NWTC) standards. ${ }^{31}$ However, the spatial variation occurs in a spanwise distance of about $10 \mathrm{~mm}$. A survey of flow quality must resolve a scale smaller than this to detect, possibly large, flow-angle gradients indicating the presence of streamwise vorticity that will create a substantial streak in the boundary layer.

The wake passing through the contraction creates the streamwise vorticity at the exit. However, there are two possible mechanisms: tilting and stretching; and Görtler instability. Contours of streamwise velocity on vertical planes through the no-slip contraction, shown in Fig. 11(a), exhibit the mushroom structure of a Görtler vortex near the contraction boundary layer, suggesting this mechanism dominates in the contraction boundary layer. At $x / L_{c}=0.6$ the Görtler vortex is less defined, and at the contraction exit it is not discernible in streamwise velocity contours. Lanspeary ${ }^{32}$ experimentally observed mushroom shaped flow structures above the boundary layer exiting the contraction of a low-speed wind tunnel. However, he deduced that the Görtler mechanism could not be responsible.

Figure 11(b) plots the maximum and minimum streamwise vorticity in the contraction for both the no-slip and slip wall contractions. Only the lower-half of the contraction and a single side of the wake symmetry $(z \geq 0)$ is considered. Streamwise vorticity cores above the layer correspond to a maximum and the vorticity of opposite rotation in the layer is a minimum. Considering the no-slip contraction case, the maximum vorticity growth in the early contraction increases along with the growth of the minimum vorticity. However, the growth of the maximum discernibly lags, and is less than, experienced by the minimum. This indicates the Görtler instability (concave region) is the dominant mechanism in this region of the contraction. The maximum begins to decay approximately $1 / 3$ rd of the way through the contraction when the wall curvature is nearly flat and stable to the Görtler instability. Again, it slightly lags the minimum in the layer. Growth begins again at approximately half way through the contraction when the local flow is stretched and tilted. Now the growth of the maximum precedes, and is significantly greater than, the growth of the minimum. The maximum vorticity in the slip-wall contraction peaks at the same streamwise location as the second peak of the no-slip contraction maximum vorticity. As the Görtler instability is not expected with the slip wall contraction, this confirms the stretching of vortex filaments is responsible for the growth of vorticity in the later part of the contraction. 
It can be deduced that in the late contraction, away from the contraction boundary layer and near-wall region, that the tilting and stretching mechanism is responsible for the creation/increasing streamwise vorticity. In the boundary layer and near-wall region of the early contraction, the Görtler instability is the primary mechanism. Streamwise vorticity in or near the contraction boundary layer can be ascribed to both mechanisms and leads to the creation of the strong cores seen in Fig. 10(a). Without the Görtler instability these cores do not appear. Far away from the contraction boundaries (Görtler influence) streamwise vorticity can be attributed to tilting and stretching. Fortunately, the strong vorticity cores did not affect the Watmuff ${ }^{21}$ experiment.

\section{COMPARISON WITH THE OPTIMAL STREAK}

Comparison with the optimal streak is made by decomposing the streaks in the spanwise direction with Fourier series

$$
Q(x, y, z)=\sum_{k=-N}^{N} \hat{Q}^{k}(x, y) e^{i k \beta z},
$$

where, $Q=[U, V, W], \hat{Q}^{k}=\left[\hat{U}^{k}, \hat{V}^{k}, \hat{W}^{k}\right]$ are the $k$ th Fourier mode, $\beta=\frac{2 \pi}{.08} \mathrm{~m}^{-1}$ for the case $2 \mathrm{~T}$ streaks, and $N$ is the number of modes in the truncated Fourier series. The spanwise wavelength of a given mode is

$$
\lambda=\frac{2 \pi}{k \beta} .
$$

The integer $k$ is used as a convenient identifier for each spanwise mode. The energy in each Fourier mode can be found by integrating to some wall-distance, arbitrarily set as $Y=6 \delta^{*}$,

$$
\hat{E}^{k}=\int_{0}^{Y}\left(\hat{U}^{k}\right)^{2}+\left(\hat{V}^{k}\right)^{2}+\left(\hat{W}^{k}\right)^{2} d y .
$$

To provide a measure of receptivity, the energy of each Fourier mode is scaled by the energy of the corresponding mode in the free-stream at some reference position, $\hat{E}_{r e f}^{k}$. The reference position is chosen as $11 \mathrm{~h}$ upstream of the leading edge where the upstream influence of the leading edge is minimal. The reference energy is defined as

$$
\hat{E}_{r e f}^{\prime k}=\left(\hat{U}_{r e f}^{k}\right)^{2}+\left(\hat{V}_{r e f}^{k}\right)^{2}+\left(\hat{W}_{r e f}^{k}\right)^{2} .
$$

Decomposing the streak into spanwise Fourier modes allows comparison with non-modal growth theory that describes the linear Optimal streak. ${ }^{1,2}$ The linear Optimal streak in the Blasius layer will reach a peak energy when the non-dimensional spanwise wavenumber $\left(\beta^{*}\right)$ of a Fourier mode is 0.74 where,

$$
\beta^{*}=k \beta \sqrt{\frac{\nu x}{U}} \equiv k \beta \delta .
$$

Similar to Fransson et al.,$^{9}$ a non-dimensional streamwise coordinate can be defined as

$$
\bar{X}=\left(\frac{k \beta \delta}{0.74}\right)^{2},
$$

so that Optimal streak would reach a maximum energy at $\bar{X}=1$.

For a given physical wavelength, $\bar{X}$ increases downstream with increasing layer thickness $(\delta)$. The streamwise position of maximum energy is used for comparison to the linear Optimal streak as it is easy to identify. The nonlinear amplitudes of the current streaks will affect mode growth. However, the streamwise shift of peak mode energy was shown by Andersson et al. ${ }^{3}$ to be small for streaks of nonlinear amplitude, generated by the Optimal streak as an initial condition. 

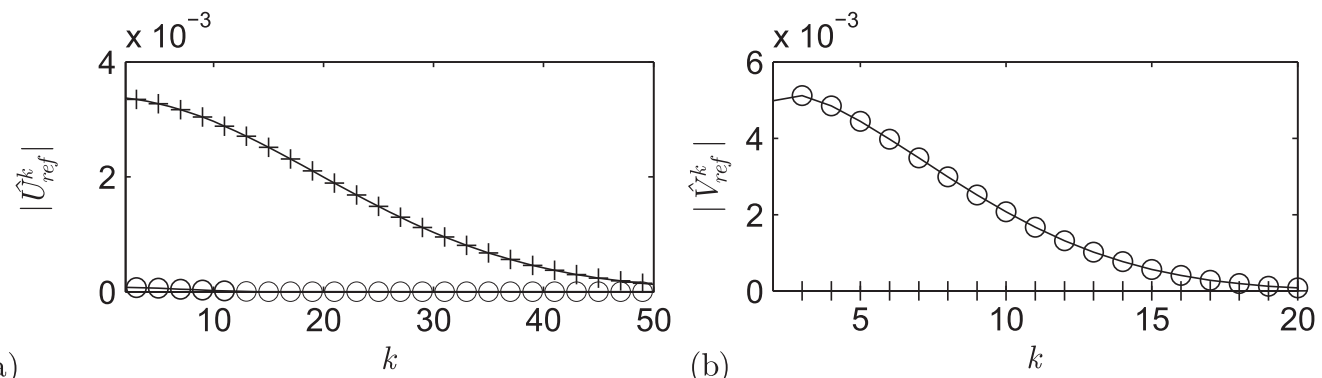

(a)

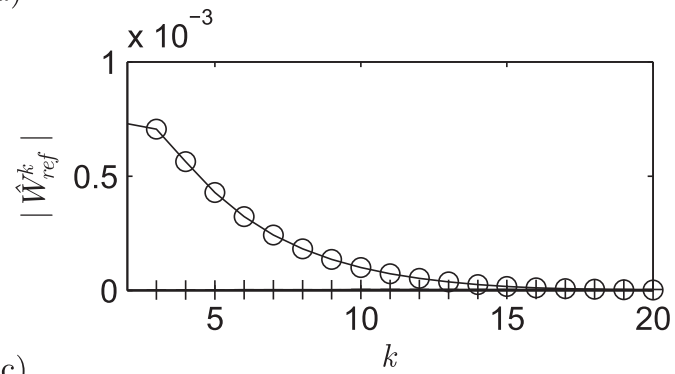

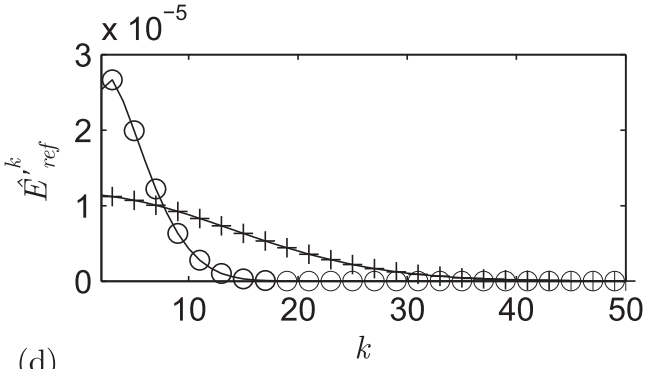

(d)

FIG. 12. FFT of the wake spanwise profile at the reference position, $11 \mathrm{~h}$ upstream of the leading edge. case2T (cross markers). case $4 \mathrm{U}$ (circle markers). (a) Streamwise velocity $\left(\mathrm{ms}^{-1}\right)$. (b) Wall-normal velocity $\left(\mathrm{ms}^{-1}\right)$. (c) Spanwise velocity $\left(\mathrm{ms}^{-1}\right)$. (d) Energy $\left(\mathrm{m}^{2} \mathrm{~s}^{-2}\right)$

\section{A. Case2T: Wire in the test-section}

Figure 12 shows the Fourier decomposition of the undisturbed case2T free-stream wake at a reference position upstream of the leading edge ( $-k$ modes are not shown due to symmetry). The majority of the wake is seen to be composed of the streamwise velocity component $(U)$ at lower wavenumbers.

The energy growth of the Fourier modes is shown in Fig. 13 where the energy is scaled by $\hat{E}_{r e f}^{\prime}$ and $\operatorname{Re}_{0.45}$, the Reynolds number when $k \beta \delta=0.45$ for a given Fourier mode. The largest wavelength to show a peak energy in the domain is the $k=5$ mode.

For the $\dot{m}_{0.17655}$ slot massflow, the peak energy of the $k=5$ mode is at $\bar{X}=0.9$, slightly upstream of the Optimal streak. Shifting the attachment point away from the leading edge centerline shifts the peak downstream and for $\dot{m}_{0.215}$ the peak energy is slightly downstream of $\bar{X}=1$. As $k$ is

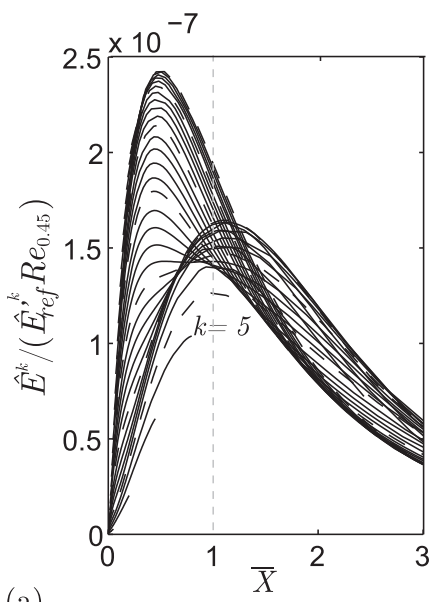

(a)

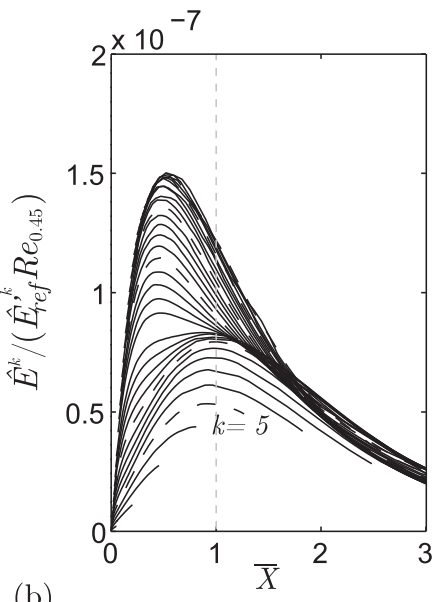

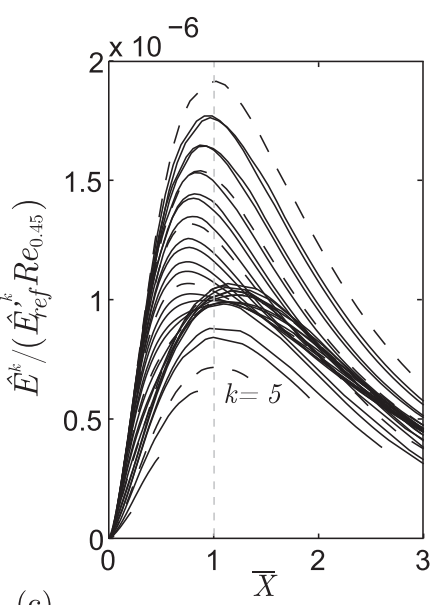

(c)

FIG. 13. Scaled Fourier mode energy for the case2T streaks versus non-dimensionalized streamwise distance (17). Fourier modes $k=1$ to 35 . Every 5 th mode is shown with dashed line. Linear Optimal streak peaks at $\bar{X}=1$. (a) $\dot{m}_{0.16}$, (b) $\dot{m}_{0.17655}$, (c) $\dot{m}_{0.215}$. 

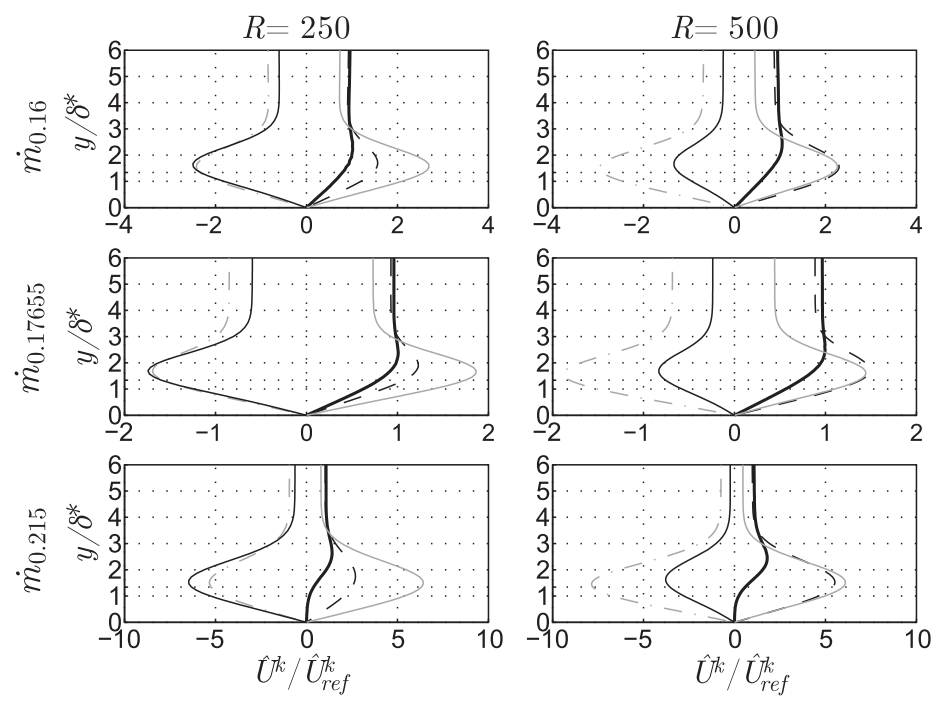

$-\mathrm{k}=1 \quad---\mathrm{k}=5$
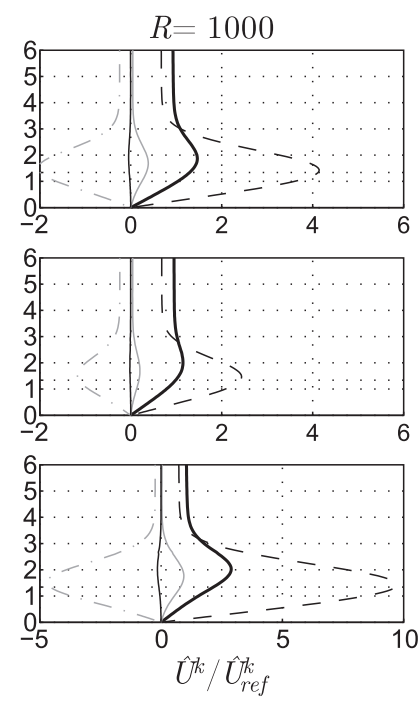

FIG. 14. Wall-normal profile of $\hat{U}^{k}$ Fourier mode shapes for the case2T streaks scaled by $\hat{U}_{r e f}^{k}$. Rows are the same slot massflow. Columns are the same $R$. Unmarked horizontal grid line is at $y / \delta^{*}=1.34$.

increased to 10 , the peak energy is seen to shift downstream to $\bar{X} \approx 1$ for $\dot{m}_{0.17655}$, and $\bar{X} \approx 1.1$ for $\dot{m}_{0.16}$ and $\dot{m}_{0.215}$. As $k$ is increased further, the peak energy then moves upstream. The $k=15$ mode exhibits a peak significantly upstream at $\bar{X} \approx 0.5$ for all slot massflows. Pressure gradient effects cannot explain this due to the differing pressure gradients for $\dot{m}_{0.16}$ and $\dot{m}_{0.215}$. Modes with $k>15$ reach $\bar{X}=1$ at $R<450$ where the layer has not yet achieved the Blasius shape factor (see Pook $^{33}$ ), making a comparison to the Optimal streak less meaningful. However, for $k \gtrsim 17$ the peak energy again shifts downstream.

Despite generating the streak from the free-stream, nonlinearity effects, and the non-Blasius base-flow in the early layer, modes with a wavelength significantly larger than $h(k \lesssim 10)$ reach their peak energy at a streamwise position near the Optimal streak. This is further downstream than for roughness generated streaks. ${ }^{9}$ The growth of steaks from normal vorticity with a wavelength significantly greater than the leading edge thickness approximates the growth of the Optimal streak.

Wall-normal mode shapes from the Fourier decomposition are shown in Fig. 14. Due to symmetry, $U^{k}$ is real. The wall-normal profile of the $k=1$ mode can be seen to vary considerably with slot massflow. At $R=250$, its maximum value is high in the boundary layer $\left(y / \delta^{*} \approx 2.5\right)$, with approximately the free-stream wake strength. For the $\dot{m}_{0.16}$ and $\dot{m}_{0.17655}$ slot massflows it reduces to zero at the wall in an approximately linear fashion. For the $\dot{m}_{0.215}$ slot massflow it reduces to near zero at a significant distance from the wall $\left(y / \delta^{*} \approx 1\right)$, giving an "s" shaped appearance. As the mode develops downstream, a peak value greater than in the wake develops high in the layer. It shifts towards the wall with increasing $R$. Large wavelength modes fail to produce substantial variation of the streamwise velocity component within the layer at low $R$. The maximum of the streamwise velocity component can actually decay with $R$ in the early layer before increasing downstream (see Pook $^{33}$ ).

The $\hat{U}^{k}$ profiles for smaller wavelengths exhibit shapes similar to the Optimal streak except they do not tend to zero above the layer due to the presence of the wake. The peak value in the layer is generally above $y / \delta^{*}=1.34$ (the location of the maximum for the Optimal streak and Klebanoff streak $u_{r m s}$ ) but is seen to shift towards $y / \delta^{*}=1.34$ with increasing $R$.

Table II documents receptivity coefficients for the $k=5$ and $k=21$ modes, defined by the streamwise maximum of $\hat{U}_{\max }^{k}$ in the layer,

$$
R c=\max \left(\frac{\left|\hat{U}_{\text {max }}^{k}\right|}{\left|\hat{U}_{r e f}^{k}\right|}\right),
$$


TABLE II. Case2T receptivity coefficient (18) for modes $k=5$ and $k=21$.

\begin{tabular}{|c|c|c|c|c|c|c|}
\hline \multirow[b]{2}{*}{$\dot{m}_{\text {slot }}$} & \multicolumn{3}{|c|}{$k=21$} & \multicolumn{3}{|c|}{$k=5$} \\
\hline & $R c$ & $\frac{R c}{r_{a}}$ & $\frac{R c}{r_{a} \cos \left(\theta_{a}\right)}$ & $\overline{R c}$ & $\frac{R c}{r_{a}}$ & $\frac{R c}{r_{a} \cos \left(\theta_{a}\right)}$ \\
\hline 0.16 & 2.8 & 32.9 & 38.1 & 4.2 & 49.4 & 57.2 \\
\hline 0.17655 & 2.0 & 51.1 & 51.1 & 2.5 & 63.9 & 63.9 \\
\hline 0.215 & 6.6 & 15.7 & 35.4 & 9.8 & 23.4 & 52.5 \\
\hline
\end{tabular}

where $\hat{U}_{r e f}^{k}$ is the free-stream value $11 h$ upstream of the leading edge. Schrader et al. ${ }^{22}$ perform a similar analysis but use the wake mode amplitude at the leading edge by extrapolating with linear theory. Unlike an energy measure integrated to some wall-distance, the amplitude maximum is unaffected by the wake above the boundary layer. The $k=21$ mode receptivity is seen to have greater variation with slot massflow relative to the $k=5$ mode. Scaling with $r_{a} \cos \left(\theta_{a}\right)$ reduces the variation of the receptivity coefficient for all slot massflows. The large wavelength $k=5$ mode receptivity is greater than the $k=21$ mode due to the greater streamwise extent of non-modal growth. The smaller variation in scaled receptivity for the $k=5$ mode indicates larger wavelength modes are less affected by the bending of streamlines approaching the attachment point, or that stretching is sensitive to the ratio of disturbance wavelength and body radius at attachment.

\section{B. Case4U: Wire upstream of the contraction}

Figure 15 shows the Fourier mode energy growth for the case $4 \mathrm{U}, \dot{m}_{0.17655}$ streak. The $\dot{m}_{0.16}$ and $\dot{m}_{0.215}$ streaks are similar and not shown (see Pook ${ }^{33}$ ). For case $4 \mathrm{U}, \beta=\frac{2 \pi}{0.2} \mathrm{~m}^{-1}$. The $k \geq 11$ modes reach $\bar{X}=1$ but are not seen to have defined peaks in the CFD domain. The $k=12$ mode for case $4 \mathrm{U}$ has a very similar wavelength to $k=5$ for case2T. Unlike the case2T $k=5$ mode that peaks near $\bar{X}=1$, the peak energy for the case $4 \mathrm{U}$ mode $k=12$ will be obtained nearer to $\bar{X}=1.5$. This is considerably downstream of the Optimal streak. Increasing $k$ for the case4U streak shifts the peak even further downstream but this may be due to the transfer of energy from larger wavelengths. The case $4 \mathrm{U}$ energy curves also exhibit a reduced growth rate in the early layer as previously noted for streak amplitude and Klebanoff streaks. ${ }^{25}$ Modes with $k \leq 10$ appear as though they will peak upstream of $\bar{X}=1$.

The receptivity coefficient for the $k=10$ mode is shown in Table III. The receptivity definition of (18) is modified by replacing $\hat{U}_{r e f}^{k}$ with $\sqrt{\hat{E}_{r e f}^{k}}$ as the case4U wake has nearly no streamwise velocity component. The case $2 \mathrm{~T}$ wake is predominately a $U$ component, allowing the receptivity coefficients to be compared. The $k=4$ mode for case2T, which has the same physical wavelength

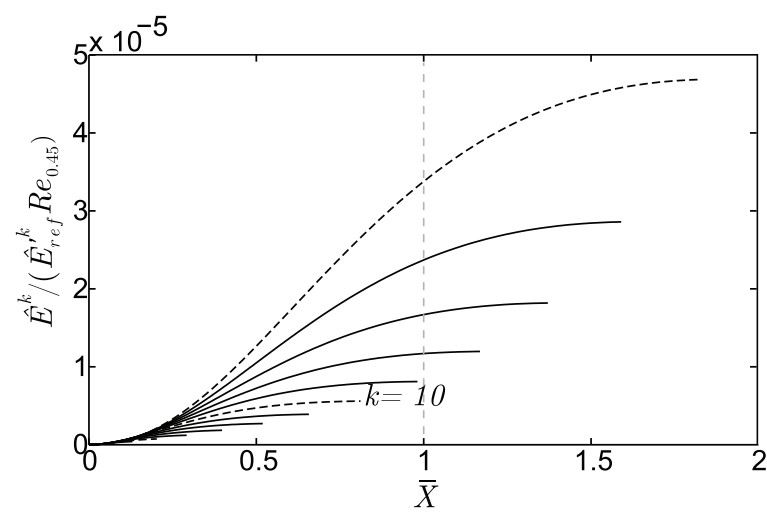

FIG. 15. Case $4 \mathrm{U}$ slot massflow $\dot{m}_{0.17655}$ scaled Fourier mode energy versus non-dimensionalized streamwise distance (17) for the Fourier modes $k=1$ to 15 . Every 5 th mode is shown with dashed line. Linear Optimal streak peaks at $\bar{X}=1$. 
TABLE III. Mode with a spanwise wavelength of $20 \mathrm{~mm}$, receptivity coefficient and streamwise location of the maximum $\left(R_{R_{c}}\right)$ for the case2T and case $4 \mathrm{U}$ streaks.

\begin{tabular}{lccccc}
\hline \hline & \multicolumn{2}{c}{ case2T, $k=4$} & & \multicolumn{2}{c}{ case4U, $k=10$} \\
\cline { 2 - 3 }$\dot{m}_{\text {slot }}$ & $R c$ & $R_{R c}$ & & $R c$ & $R_{R c}$ \\
\hline 0.16 & 4.4 & 1430 & 32 & 1120 \\
0.17655 & 2.5 & 1400 & & 31 & 1160 \\
0.215 & 10.5 & 1350 & & 28.7 & 1250 \\
\hline \hline
\end{tabular}

$(20 \mathrm{~mm})$, is also listed along with the streamwise position of the maximum $\left(R_{R c}\right)$. Considering the same slot massflow, $\dot{m}_{0.17655}$, the case2T streak $\left|\hat{U}_{\max }^{k}\right|$ is found to peak downstream of the case $4 \mathrm{U}$ streak. It should be noted that the streamwise maximum $\left|\hat{U}_{\max }^{k}\right|$ does not correspond with the streamwise maximum of energy. The case $4 \mathrm{U}$ streak receptivity coefficient is 12.4 times larger. For $\dot{m}_{0.215}$, the difference is reduced to a factor of 2.7. As a comparison, Schrader et al. ${ }^{22}$ found an approximate factor of 5 for a modified super-elliptic leading edge with an aspect ratio of 6 when using steady disturbances that maintain linear amplitudes. The experiment of Watmuff ${ }^{21}$ provides experimental evidence that the boundary layer is generally more receptive to streamwise vorticity, but the receptivity to normal vorticity can be increased substantially by modifying the attachment position.

Wall-normal Fourier mode shapes for the case4U streak are plotted in Fig. 16. The $\hat{U}^{k}$ profiles at $R=250$ for all modes except $k=20$ exhibit a peak amplitude near $y / \delta^{*}=1.34$. With increasing streamwise distance, the peak of the $k=1$ mode moves away from the wall and peaks near $y / \delta^{*}=$ 2.5. The $k=15$ and $k=20$ modes show a similar trend. The $k=5$ and $k=10$ modes peaks shift very slightly with streamwise distance, maintaining a profile close to the Optimal streak and Klebanoff streak $u_{r m s}$. The $k=20$ mode is visibly inflectional near the wall for $R=500$ and $R=1000$, while a slightly inflectional profile can be seen for the $k=15$ mode at $R=1000$. The Fourier mode profiles for the case2T streak did not develop inflections (see Fig. 14). However, while individual Fourier modes are inflectional for the case $4 \mathrm{U}$ streak, no velocity profile through the streak is found to be inflectional. With increased streamwise distance and streak strength, it seems likely that more Fourier modes could develop an inflection point which would lead to inflectional velocity profiles at some spanwise locations. The analysis of Goldstein et al. ${ }^{17}$ and Goldstein and Leib ${ }^{18}$ found that a streak created by streamwise vorticity would develop an inflectional velocity profile away from the streak centerline prior to separation, while a streak created by normal vorticity would separate on the streak centerline first.

The significant energy growth beyond $\bar{X}=1$ for smaller wavelength Fourier modes, and the reduced growth rate in the early layer for streamwise vorticity generated streaks, suggests the
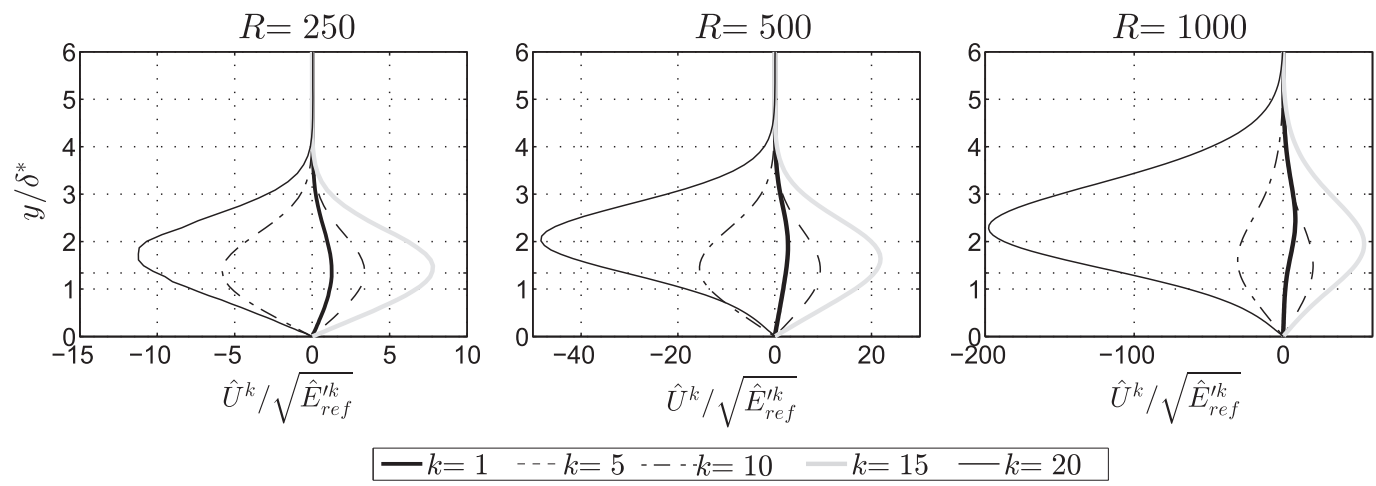

FIG. 16. Wall-normal profile of $\hat{U}^{k}$ Fourier mode shapes (real component) for the case $4 \mathrm{U} \dot{m}_{0.17655}$ streak, scaled by $\sqrt{\hat{E}_{\text {ref }}^{\prime}}$. Unmarked horizontal grid line is at $y / \delta^{*}=1.34$. 
growth of the Optimal streak may not be an ideal model for Klebanoff streaks. This assumes that Klebanoff streaks are generated by free-stream streamwise vorticity, which the combined results of Kendall, ${ }^{27}$ Watmuff, ${ }^{28}$ Schrader et al. ${ }^{22}$ and this paper indicates. Due to the amplitude of the case $4 \mathrm{U}$ streak, nonlinearity will affect individual mode growth. This may be the cause of the large $k$ modes growing beyond $\bar{X}=1$ and small $k$ modes peaking upstream. Another important factor is the relative distribution of the initial free-stream modes, i.e., the free-stream disturbance profile. The current streak is generated by a Gaussian wake, a universal wake profile for a single body, passing through a contraction and it suggests the growth of individual modes in the streak can vary substantially compared to the Optimal. Different streak growth may have significant implications for the effect of the streak on TS growth and streak secondary instability.

\section{DISCUSSION AND CONCLUSION}

The CFD results have reproduced the experimental streaks of Watmuff ${ }^{21}$ to good accuracy and provide physical, reproducible numbers that contribute to the study of flow quality issues and testsection boundary layer spanwise variation. Reproducing the results allows the original experiment to be viewed as a receptivity experiment. The receptivity to streamwise vorticity is shown to be considerably greater than normal vorticity for a sharp leading edge, which has also been found computationally by Schrader et al. ${ }^{22}$ The relative receptivity can be varied significantly by the leading edge attachment position and the corresponding bluntness. The results showed the ratio of streamwise receptivity coefficient to normal vorticity varied between factors of 2.5 and 12.4 for a $20 \mathrm{~mm}$ wavelength (results include nonlinear effects).

This highlights the attachment position on the leading edge as an important receptivity issue. Many experiments alter the flow attachment position to reduce the adverse pressure gradient in the recovery region. However, simulations often assume attachment on the leading edge centerline. The order of magnitude variation in normal vorticity streak strength with attachment position highlights this as an important issue for any computation that seeks to recreate experimental data that involve the receptivity of normal vorticity.

Spanwise Fourier decomposition of the streaks allowed energy growth to be compared with the linear Optimal streak. For the case2T streaks, modes with a spanwise wavelength significantly larger than the leading edge thickness show a streamwise peak close to where the Optimal would peak. Wavelengths of the order of the leading edge thickness exhibit a peak energy considerably upstream of the Optimal that varies substantially with attachment position. This suggests that experimental streaks with similar behavior to the Optimal could be generated by wakes of normal vorticity with a wavelength significantly larger than the leading edge thickness. However, the case $2 \mathrm{~T}$ streaks can exhibit $\hat{U}^{k}$ wall-normal profiles with peak values slightly higher than $y / \delta^{*}=1.34$. The two streamwise peaks of streak amplitude can be explained by the narrow free-stream wake of the test-section wire, and the increased receptivity to normal vorticity with a wavelength similar to the leading edge thickness that reaches a maximum energy upstream of $\bar{X}=1$. A modified spanwise wake profile, with relatively stronger large wavelength modes, could produce a streak with a large extent of streamwise growth.

For the case $4 \mathrm{U}$ streaks, the streamwise peak energy shifts downstream with decreasing wavelength, possibly due to energy transfer from larger wavelengths. Larger wavelength modes appear as though they would peak upstream of $\bar{X}=1$ if the CFD domain was extended. The energy growth rate was also reduced in the early layer $(\bar{X}<0.25)$. Examination of the Fourier mode wall-normal profiles revealed mode shapes considerably different to the Optimal streak for smaller wavelengths beyond $\bar{X}=1$, particularly downstream when inflection points become visible. However, a wallnormal inflection point was not visible on a given profile through the streak. The $k=5$ and $k=$ 10 modes exhibited a mode shape similar to the Optimal through the entire domain $(\bar{X} \leq 1)$. It is suggested that the growth of the Optimal streak is different to the growth of the streamwise vorticity generated streak (case $4 \mathrm{U}$ ), and growth of this streak is different to the growth of a streak generated from normal vorticity (case2T).

The recent computations of Vaughan and $\mathrm{Zaki}^{19}$ demonstrated a streak generated by a Squire mode has considerably different linear stability characteristics to the Optimal streak, indicating 
an accurate model must be used for Klebanoff streaks in transition studies. The experiments of Kendall $^{27}$ and Watmuff ${ }^{28}$ exhibited Klebanoff streaks that were insensitive to the leading edge, indicating they were generated by streamwise vorticity. The case $4 U$ wire is generated by free-stream streamwise vorticity from a wake that passes through a contraction. It is suggested that this can be a dominant mechanism in the creation of Klebanoff streaks, where the wake of a single wire used in the current study is replaced by the wake of a settling chamber screen. This indicates the possibility that Klebanoff streaks generated by screens upstream of the contraction (streamwise vorticity) and Klebanoff streaks generated by a grid in the test-section (possibly dominated by normal vorticity) may have different growth and stability characteristics. Supporting evidence for the generation of Klebanoff streaks by the tilting and stretching of normal vorticity in a contraction can be found in the observations of de Bray, ${ }^{34}$ who observed the spanwise variation of skin-friction on a test-section plate mounted on the wind tunnel centerline could be halved relative to the tunnel walls. At the very least, the current CFD results describe in detail the important parameters dictating streak generation in the experiment of Watmuff, ${ }^{21}$ allowing the design of future experiments with streaks generated from free-stream disturbances.

The efficacy of the contraction in turning normal vorticity into streamwise vorticity (60\% at the leading edge for the current results), and the significantly increased receptivity of the layer to streamwise vorticity (order of magnitude greater for the sharp leading edge), suggests an interesting mechanism to explain the generation of Klebanoff streaks in wind tunnels. Clearly, the contraction must be considered a vital component affecting spanwise variation of the downstream boundary layer despite increasing uniformity of the free-stream, streamwise velocity component. Contractions are often associated with the stretching of streamwise vorticity entering the contraction (greatest on the centerline). Hence, streamwise vorticity can be expected over the entire tunnel cross-section if normal and streamwise vorticity are present upstream of the contraction. Any survey of flow quality in the test-section must be able to measure weak but concentrated crossflow velocity components and must be done with a spanwise resolution sufficiently small to detect the gradients of crossflow velocities. The current results for a flow velocity of approximately $9.5 \mathrm{~ms}^{-1}$, show that flow angles less than $0.5^{\circ}$ in the leading edge region and concentrated in a spanwise distance of approximately 10 $\mathrm{mm}$, produced a streak of substantial strength. A flow quality survey to detect streamwise vorticity causing Klebanoff streaks must resolve amplitudes and distances smaller than this. A flow quality survey could be designed using non-modal growth theory ${ }^{1,2}$ to predict wavelengths that will peak in a given streamwise domain of interest and the presented receptivity data, and that of Schrader et al. ${ }^{22}$ can be used to approximately relate that to a free-stream disturbance amplitude (flow angle) that must be detected.

\section{ACKNOWLEDGMENTS}

D.P. acknowledges funding from the Australian Postgraduate Award (APA). J.W. acknowledges funding from the Defence Scientific and Technical Organisation (DSTO) via the research Agreement No. 2009/1107597/11. Access to the Trifid HPC cluster provided by the V3 Alliance and the assistance of the RMIT eResearch Office is gratefully acknowledged.

\footnotetext{
${ }^{1}$ P. Andersson, M. Berggren, and D. S. Henningson, “Optimal disturbances and bypass transition in boundary layers," Phys. Fluids 11(1), 134-150 (1999).

${ }^{2}$ P. Luchini, "Reynolds-number-independent instability of the boundary layer over a flat surface: Optimal perturbations," J. Fluid Mech. 404, 289-309 (2000).

${ }^{3}$ P. Andersson, L. Brandt, A. Bottaro, and D. S. Henningson, “On the breakdown of boundary layer streaks," J. Fluid Mech. 428, 29-60 (2001).

${ }^{4}$ C. Cossu and L. Brandt, "Stabilization of Tollmien-Schlichting waves by finite amplitude optimal streaks in the Blasius boundary layer," Phys. Fluids 14(8), 1-4 (2002).

${ }^{5}$ L. Brandt, C. Cossu, J. Chomaz, P. Huerre, and D. S. Henningson, "On the convectively unstable nature of optimal streaks in boundary layers," J. Fluid Mech. 485, 221-242 (2003).

${ }^{6}$ C. Cossu and L. Brandt, "On Tollmien-Schlichting-like waves in streaky boundary layers," Europ. J. Mech. B/Fluids 23(6), 815-833 (2004).

${ }^{7}$ S. Bagheri and A. Hanifi, "The stabilizing effect of streaks on Tollmien-Schlichting and oblique waves: A parametric study,” Phys. Fluids 19, 19-22 (2007).
} 
${ }^{8}$ P. Schlatter, E. Deusebio, R. de Lange, and L. Brandt, Numerical Study Of The Stabilisation Of Boundary-Layer Disturbances By Finite Amplitude Streaks, Swedish e-Science Research Centre, Linne FLOW Centre, KTH Mechanics, Stockholm, Sweden, 2011

${ }^{9}$ J. H. M. Fransson, L. Brandt, A. Talamelli, and C. Cossu, "Experimental and theoretical investigation of the nonmodal growth of steady streaks in a flat plate boundary layer," Phys. Fluids 16(10), 3627-3638 (2004).

${ }^{10}$ J. H. M. Fransson, L. Brandt, A. Talamelli, and C. Cossu, "Experimental study of the stabilization of Tollmien-Schlichting waves by finite amplitude streaks," Phys. Fluids 17(5), 054110 (2005).

${ }^{11}$ J. H. M. Fransson, A. Talamelli, L. Brandt, and C. Cossu, "Delaying transition to turbulence by a passive mechanism," Phys. Rev. Lett. 96(6), 064501 (2006).

${ }^{12}$ F. P. Bertolotti and J. M. Kendall, "Response of the Blasius boundary layer to controlled free-stream vortices of axial form," AIAA Paper No. 97-2018, 1997.

${ }^{13}$ A. V. Boiko, "Flat-plate boundary layer receptivity to a steady free-stream vortex disturbance," Fluid Dyn. 36(6), 915-925 (2001).

${ }^{14}$ A. V. Boiko and H. H. Chun, "Development of low-frequency streaks in Blasius boundary layer," Phys. Fluids 16(8), 3153-3160 (2004).

${ }^{15}$ J. H. M. Fransson and A. Talamelli, "On the generation of steady streamwise streaks in flat-plate boundary layers," J. Fluid Mech. 698, 211-234 (2012).

${ }^{16}$ S. Shahinfar, S. S. Sattarzadeh, J. H. M. Fransson, and A. Talamelli, "Revival of classical vortex generators now for transition delay," Phys. Rev. Lett. 109(7), 074501 (2012).

${ }^{17}$ M. E. Goldstein, S. J. Leib, and S. J. Cowley, "Distortion of a flat-plate boundary layer by free-stream vorticity normal to the plate," J. Fluid Mech. 237, 231-260 (1992).

${ }^{18}$ M. E. Goldstein and S. J. Leib, "Three-dimensional boundary-layer instability and separation induced by small-amplitude streamwise vorticity in the upstream flow," J. Fluid Mech. 246, 21-41 (1993).

${ }^{19}$ N. J. Vaughan and T. A. Zaki, "Stability of zero-pressure-gradient boundary layer distorted by unsteady Klebanoff streaks," J. Fluid Mech. 681, 116-153 (2011).

${ }^{20}$ M. N. Kogan, V. G. Shumilkin, M. V. Ustinov, and S. V. Zhigulev, "Response of boundary layer flow to vortices normal to the leading edge," Europ. J. Mech. B/Fluids 20(6), 813-820 (2001).

${ }^{21}$ J. H. Watmuff, "Effects of weak free stream nonuniformity on boundary layer transition," J. Fluids Eng. 128(2), 247-257 (2006).

${ }^{22}$ L. Schrader, L. Brandt, C. Mavriplis, and D. S. Henningson, "Receptivity to free-stream vorticity of flow past a flat plate with elliptic leading edge," J. Fluid Mech. 653, 245-271 (2010).

${ }^{23}$ G. K. Batchelor, An Introduction to Fluid Dynamics, 2nd ed. (Cambridge University Press, 2000); L. D. Landau and E. M. Lifshitz, Fluid Mechanics: Course of Theoretical Physics, 2nd ed. (Butterworth-Heinemann Ltd, 2008), Vol. 6; H. Schlichting and K. Gersten, Boundary-Layer Theory, 8th ed. (Springer, 2003).

${ }^{24}$ S. Sen, S. Mittal, and G. Biswas, "Steady separated flow past a circular cylinder at low Reynolds numbers," J. Fluid Mech. 620, 89-119 (2009).

${ }^{25}$ J. H. M. Fransson and P. H. Alfredsson, "Transition induced by free-stream turbulence," J. Fluid Mech. 527, 1-25 (2005).

${ }^{26}$ K. J. A. Westin, A. V. Boiko, B. G. B. Klingmann, V. V. Kozlov, and P. H. Alfredsson, "Experiments in a boundary layer subjected to free stream turbulence. Part 1. Boundary layer structure and receptivity," J. Fluid Mech. 281, 193-218 (1994)

${ }^{27}$ J. M. Kendall, "Experiments on boundary-layer receptivity to freestream turbulence," AIAA Paper No. 98-0530, 1998.

${ }^{28}$ J. H. Watmuff, "Detrimental effects of almost immeasurably small freestream nonuniformities generated by wind-tunnel screens," AIAA J. 36(3), 379-386 (1998).

${ }^{29}$ M. V. Ustinov, "Response of the boundary layer developing over a blunt-nosed flat plate to free-stream non-uniformities," Europ. J. Mech. B/Fluids 20(6), 799-812 (2001).

${ }^{30}$ S. J. Kline, Film Notes for Flow Visualization (National Committee for Fluid Mechanics Films, 1969), pp. 1-5.

${ }^{31}$ F. K. Owen and A. K. Owen, "Measurement and assessment of wind tunnel flow quality," Prog. Aerospace Sci. 44(5), 315-348 (2008).

${ }^{32} \mathrm{P}$. V. Lanspeary, "Establishing very low speed, disturbance-free flow for anemometry in turbulent boundary layers," $\mathrm{Ph} . \mathrm{D}$ thesis (University of Adelaide, 1997).

${ }^{33}$ D. A. Pook, "The origin and effects of free-stream vortical disturbances on boundary layer transition in wind tunnels," Ph.D. thesis (RMIT University, 2013).

${ }^{34}$ B. G. de Bray, "Some investigations into the spanwise non-uniformity of nominally two-dimensional incompressible boundary layers downstream of gauze screens," Reports and Memoranda No. 3578, Aeronautical Research Council, 1967. 